\title{
Pancreatitis Can Present Like Cancer: Lymphoplasmacytic Sclerosing Pancreatitis in a Patient with a History of Gastric Carcinoma
}

\author{
Susana Rocha Amaral ${ }^{1}$, Luís Elvas ${ }^{2}$, Tatiana Pereira ${ }^{1}$, Paula Jacinto ${ }^{1 .}$ Gabriela Sousa $^{1}$ \\ ${ }^{1}$ Medical Oncology Department, Instituto Português de Oncologia de Coimbra, Coimbra, Portugal \\ ${ }^{2}$ Gastroenterology Department, Instituto Português de Oncologia de Coimbra, Coimbra, Portugal
}

Received: 09/07/2019

Accepted: 25/07/2019

Published: 06/08/2019

\begin{abstract}
How to cite this article: Rocha Amaral S, Elvas L, Pereira T, Jacinto P, Sousa G. Pancreatitis can present like cancer: lymphoplasmacytic sclerosing pancreatitis in a patient with a history of gastric carcinoma. EJCRIM 2019;6: doi:10.12890/2019_001205.
\end{abstract}

Conflicts of Interests: The Authors declare that there are no competing interest

Acknowledgements: We thank Dr. Paulo Figueiredo, Head of Department of Anatomical Pathology of Instituto Português de Oncologia de Coimbra, for providing the pathology images.

This article is licensed under a Commons Attribution Non-Commercial 4.0 License

\section{ABSTRACT}

Autoimmune pancreatitis has been associated with many syndromes in the presence of increased immunoglobulin levels. IgG4 antibodies are elevated in the context of lymphoplasmacytic sclerosing pancreatitis associated with IgG4-related disease. We present the case of a 74-year-old man diagnosed with autoimmune pancreatitis on a cancer background. Awareness of this condition in the cancer patient is crucial for timely diagnosis. Infectious complications might have implications for the choice of immunosuppressant.

\section{LEARNING POINTS}

- Autoimmune pancreatitis can mimic pancreatic cancer, thus delaying diagnosis in patients with cancer.

- It is important to rule out malignancy and distinguish autoimmune pancreatitis from pancreatic cancer, but obtaining a sample of pancreatic tissue can be difficult.

- Steroids are the mainstay of initial treatment, but there is growing evidence for the use of other immunomodulators, such as rituximab, for induction and maintenance or as an option for patients at high risk of relapse.

\section{KEYWORDS}

Autoimmune pancreatitis, lymphoplasmacytic sclerosing pancreatitis, IgG4-related disease, medical oncology

\section{INTRODUCTION}

Autoimmune pancreatitis (AIP) is a chronic fibroinflammatory disease of the pancreas that belongs to the spectrum of immunoglobulin G-subclass 4-related diseases (IgG4-RD) ${ }^{[1]}$. Lymphoplasmacytic sclerosing pancreatitis (LPSP) typically presents with painless obstructive jaundice $^{[1,2]}$. Pancreatic imaging, measurement of serum IgG4 levels, and determination of other organ involvement are all helpful for distinguishing AIP from pancreatic cancer ${ }^{[3,4]}$. LPSP is highly responsive to steroids, but immunomodulators should be included in induction therapy in patients who fulfil the severity criteria ${ }^{[2]}$.

\section{CASE DESCRIPTION}

A 74-year-old Caucasian man presented to the outpatient clinic in May 2018 with painless obstructive jaundice. He had a history of stage IIA gastric cancer in 2013 (submitted to perioperative chemotherapy with six cycles of cisplatin+5-fluorouracil and total gastrectomy with complete pathological response) and also of non-muscle-invasive bladder cancer in 2017 (submitted to trans-urethral resection). Colangio-MRI was performed and showed a mass measuring $40 \times 30 \times 40 \mathrm{~mm}$ in the head of the pancreas and causing obstruction of the main pancreatic and biliary duct leading to dilatation of the biliary tree (Fig. 1A). Tumour markers (CEA, CA19.9) were normal. IgG4 levels were not 
measured. A percutaneous pancreatic biopsy was performed since endoscopic ultrasonography was not feasible due to the surgical history. The specimen did not show any histological changes compatible with malignancy or inflammatory disease. A biliary stent was inserted. The patient was discharged with clinical and imaging re-evaluation scheduled within the following 3 months.

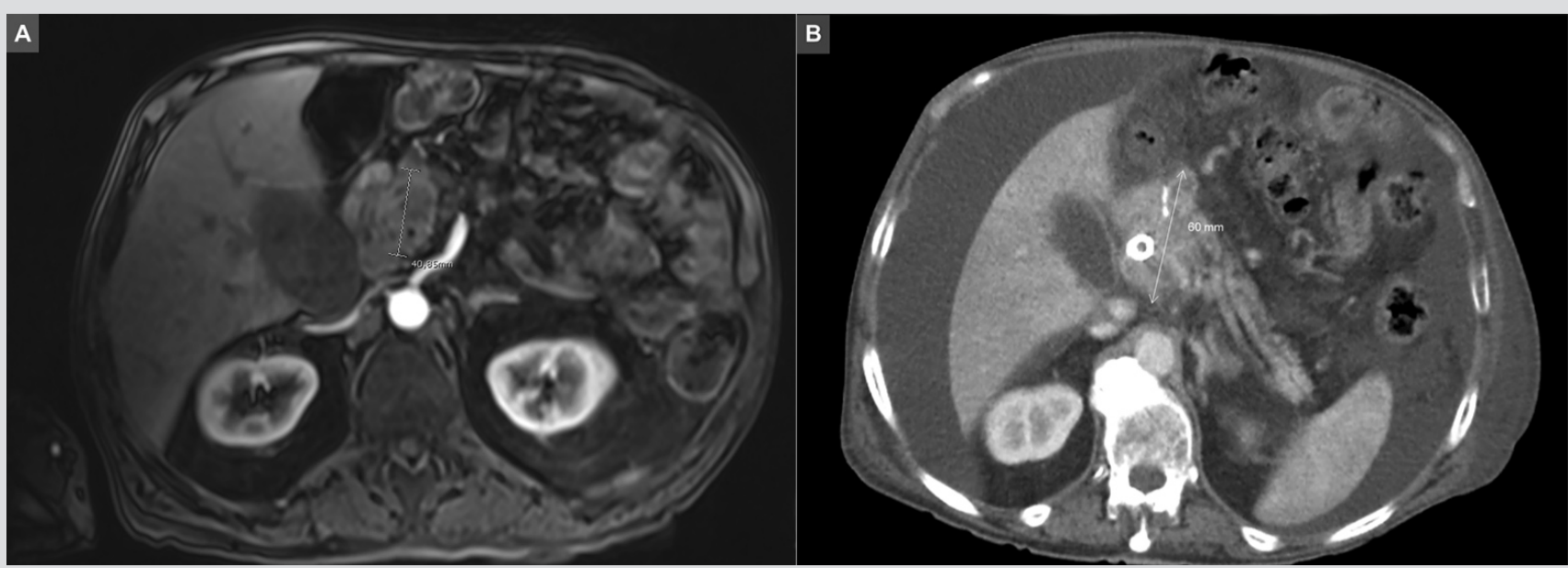

Figure 1. Radiologic features of pancreatic lesion. - A - Colangio magnetic resonance imaging (CMRI) confirms the presence of a heterogeneous mass in the head of the pancreas, with decreased signal intensity on T1 weighted image. B - Computed tomographic (CT) scan shows a diffusely enlarged pancreas with a heterogeneous mass of about $60 \mathrm{~mm}$ of maximum dimeter in the head of the pancreas.

Shortly before the date of re-evaluation, the patient was admitted to hospital because of new-onset ascites, peripheral oedema and acute kidney injury. He was pale, thin and dehydrated, with a distended abdomen without palpable masses, and oedema below the knees. The laboratory work-up revealed normocytic/normochromic anaemia, hypoalbuminaemia, normal bilirubinaemia, increased alkaline phosphatase and aminotransferases, increased IgG4, acute kidney injury and hyponatraemia (Table 1). Diagnostic paracentesis showed no signs of infection or malignancy. The abdominal CT scan showed an increase in the size of the pancreatic mass and portal vein thrombosis (Fig. 1B). Percutaneous biopsy was repeated and showed lymphoplasmacytic foci, marked storiform fibrosis, CD138 positivity and IgG4 expression (Fig. 2).

\begin{tabular}{|c|c|c|c|c|c|}
\hline Variable & Reference range & $\begin{array}{l}\text { 1st Hospital } \\
\text { admission }\end{array}$ & $\begin{array}{l}\text { After } 4 \text { weeks } \\
\text { of steroids }\end{array}$ & After tapering & $\begin{array}{l}\text { 2nd Admission, } \\
\text { day } 12\end{array}$ \\
\hline $\begin{array}{c}\text { Haemoglobin } \\
(\mathrm{g} / \mathrm{dL})\end{array}$ & $13-18$ & 9.6 & 11.5 & 13.8 & 13.0 \\
\hline Platelets $\left(\mathrm{mm}^{3}\right)$ & $150,000-400,000$ & 190,000 & 267,000 & 114,000 & 166,000 \\
\hline Leucocytes $\left(\mathrm{mm}^{3}\right)$ & $4.500-11,000$ & 11,400 & 11,500 & 11,800 & 12,300 \\
\hline Urea (mg/dl) & $10-50$ & 27 & 14 & 75 & 137 \\
\hline Creatinine $(\mathrm{mg} / \mathrm{dl})$ & $0.5-1.2$ & 2.73 & 0.8 & 1.4 & 2.4 \\
\hline Sodium (mmol/l) & $135-150$ & 133 & 139 & 131 & 133 \\
\hline $\begin{array}{l}\text { Potassium } \\
(\mathrm{mmol} / \mathrm{l})\end{array}$ & $3.5-5.3$ & 4.8 & 4.0 & 4.7 & 5.5 \\
\hline Albumin (g/dl) & $3.5-5.0$ & 2.0 & 3.1 & 2.7 & 2.7 \\
\hline Bilirubin (mg/dl) & $\leq 1.0$ & 0.6 & 1.0 & 0.7 & 0.9 \\
\hline $\operatorname{ALP}(U / I)$ & $\leq 117$ & 246 & 108 & 156 & 181 \\
\hline AST (U/I) & $\leq 38$ & 89 & 24 & 42 & 49 \\
\hline $\operatorname{ALT}(\mathrm{U} / \mathrm{I})$ & $\leq 41$ & 59 & 19 & 27 & 25 \\
\hline GGT (U/I) & $11-49$ & 547 & 228 & 187 & 171 \\
\hline INR & $\leq 1.20$ & 1.69 & 1.33 & 1.82 & 1.63 \\
\hline $\lg G 4$ & $39.2-864.0$ & 2248 & 1379.5 & 952.7 & - \\
\hline
\end{tabular}

Table 1. Laboratory findings 


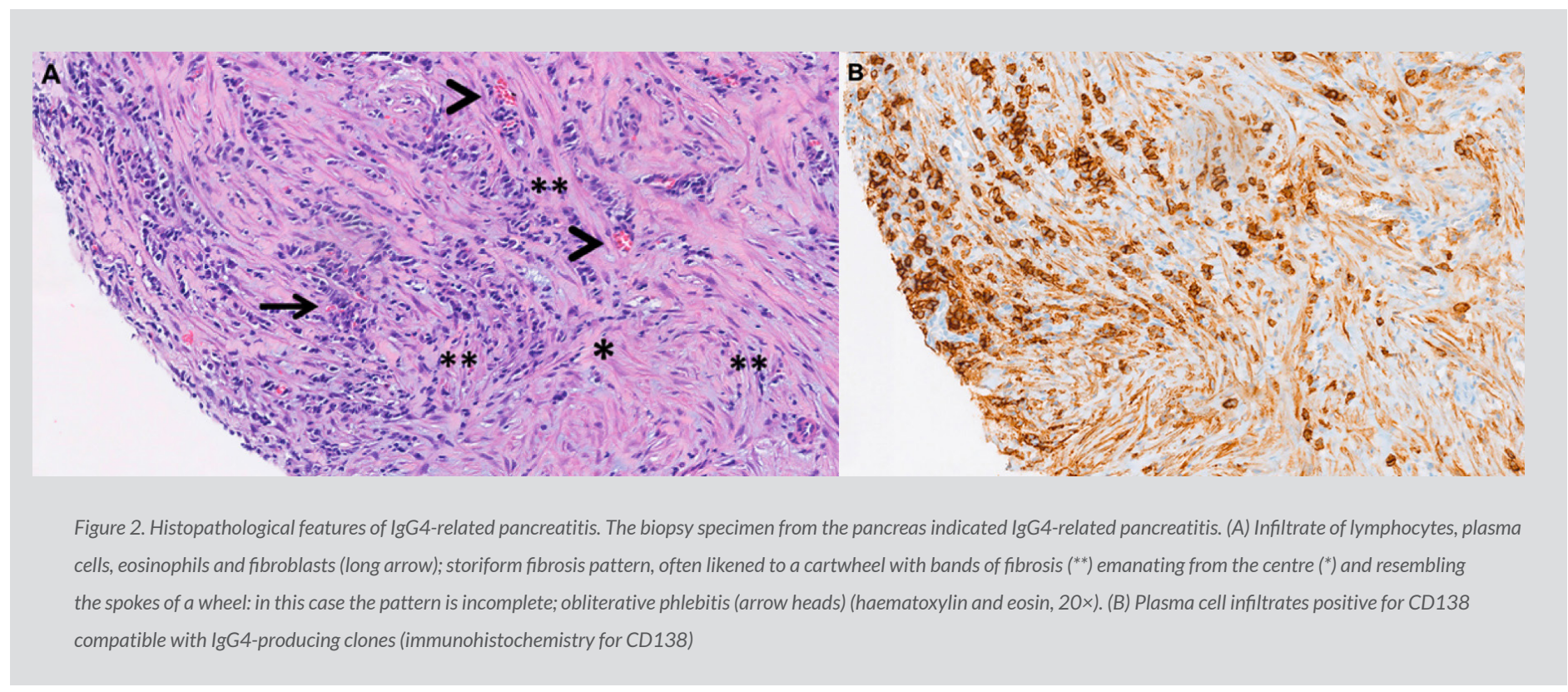

The diagnosis of LPSP was established and the patient started treatment with steroids ( $40 \mathrm{mg} / \mathrm{day}$ ) and anticoagulation for the portal vein thrombosis. During his hospital admission, the patient developed Escherichia coli bacteraemia that first manifested as cholangitis, which required intravenous antibiotics. The patient responded partially to the treatment (his lgG4 level dropped to 1379) and a first attempt at steroid tapering was made. However, the patient deteriorated clinically and had to be readmitted to hospital. The dose of steroids was increased to $40 \mathrm{mg} / \mathrm{day}$ with no improvement. The patient's clinical situation rapidly declined due to severe sepsis which started as another episode of cholangitis. There was no response to broad-spectrum intravenous antibiotics and the patient died on day 12.

\section{DISCUSSION}

LPSP is the pancreatic manifestation of systemic IgG4-RD. The typical patient with LPSP is over 60-70 years of age, with male predominance $(3: 1)^{[2]}$. Other clinical presentations include a pancreatic mass on imaging, focal or diffuse pancreatic enlargement, pancreatic ductal strictures and rarely, acute pancreatitis ${ }^{[2]}$. The clinical presentation can mimic other types of pancreatitis and pancreatic cancer ${ }^{[4]}$. The incidence of malignancy in patients with IgG4-RD is similar to that observed in the general population. However, the incidence of AIP in patients with cancer is unknown and hard to establish particularly because of the difficulties of diagnosis.

Patients with LPSP most commonly present with a cholestatic pattern of liver enzyme elevation and raised lgG4 levels (>2 times the upper limit of normal). The diagnosis of LPSP is based on histological and imaging findings. The key histological finding is lymphoplasmacytic infiltration rich in IgG4-positive plasma cells combined with storiform fibrosis and obliterative phlebitis ${ }^{[5]}$. Imaging studies show a diffusely enlarged gland with featureless borders and delayed enhancement with/without a capsule-like rim ${ }^{[2]}$. In our case, the diagnosis was delayed mainly due to lack of awareness of this condition and the high probability of malignancy. Consequently, treatment with steroids was delayed for 3 months when there was decompensated cholestatic hepatic disease.

Steroids are a cornerstone in the management of LPSP. Most patients show remarkable initial improvement with prednisone administration as shown by a decrease in liver enzymes. The recommended regimen is prednisone $40 \mathrm{mg} /$ day for 4 weeks, and slow tapering by $5 \mathrm{mg} / \mathrm{week}$ after evaluation of clinical, radiological and serological response. Other immunosuppressant therapy, such as rituximab, might be considered for induction in patients with risk factors for relapse: proximal biliary involvement, younger age and high alkaline phosphatase. Our patient had two criteria for the use of rituximab and his disease was refractory to therapy with steroids. However, infectious complications precluded the use of this B-cell depletion therapy.

The diagnosis of LPSP is difficult, particularly in the cancer patient, where the diagnostic assessment is sometimes simplified and the therapeutic intervention tailored for symptom management. Higher awareness of the diagnosis could have resulted in earlier introduction of steroid therapy in our patient. Stratifying the risk for relapsing disease is mandatory. Infectious complications commonly limit therapeutic intervention in these patients. 


\section{REFERENCES}

1. Majumder S, Takahashi N, Chari ST. Autoimmune pancreatitis. Dig Dis Sci 2017; 62 (7):1762-1769.

2. Nagpal SJS, Sharma A, Chari ST. Autoimmune pancreatitis. Am J Gastroenterol 2018;113(9):1301.

3. Chari ST, Takahashi N, Levy MJ, Smyrk TC, Clain JE, Pearson RK, et al. A diagnostic strategy to distinguish autoimmune pancreatitis from pancreatic cancer. Clin Gastroenterol Hepatol 2009;7(10):1097-1103.

4. Marinho R, Alves A, Pignatelli N, Nunes V. Unclassified autoimmune pancreatitis mimicking pancreatic cancer. J Surg Case Rep 2019;2019(1):rjy340.

5. Detlefsen S, Kloppel G. IgG4-related disease: with emphasis on the biopsy diagnosis of autoimmune pancreatitis and sclerosing cholangitis. Virchows Arch 2018;472(4):545556. 